25.6. - Nürnberg: Praxis-Seminar: Diagnostik und Therapie des Kopfschmerzes. Anmeldung: siehe 16.1 .

2.-4.9. - Bremen: IV. Internationales Symposium über Schmerzdiagnostik und -therapie des Roten-Kreuz-Krankenhauses Bremen. Auskunft: siehe 2.4.

7.-8.10. - Basel: 13. Jahrestagung der Gesellschaft zum Studium des Schmerzes für Deutschland, Österreich und die Schweiz. Thema: Chronischer Schmerz und Psyche. Diagnostische und therapeutische Modelle. Auskunft: Kongreß-Sekretariat, Postfach 29, CH-4005 Basel

10.9. - Nürnberg: Praxis-Seminar: Diagnostik und Therapie des Kreuzschmerzes. Anmeldung: siehe 16.1 .
10.-14.9. - Florenz: 3rd International Symposium ,The Pain Clinic“. Auskunft: Prof. M. Zoppi, CESPRI Fondazione Pro Juventute, Via Imprunetana 124, I-50020 Monteoriolo, Florenz, Tel. 055/208322-426

16.-17.9. - Hamburg: Praxis-Seminar: Psychotherapeutische Schmerzbehandlung mit den Methoden des NLP I (Neurolinguistisches Programmieren). Anmeldung: siehe 5.-6. 2 .

30.9.-1.10. - Frankfurt: Praxis-Seminar: Lokal- und Leitungsanästhesie in der Schmerztherapie. Anmeldung: siehe 18.19.3 .

5.10. - Aachen: Praxis-Seminar: Diagnostik und Therapie des Brustschmerzes. Anmeldung: siehe 18.5.
28.-29.10. - Hamburg: Praxis-Seminar: Diagnostik und Therapie des Tumorschmerzes. Anmeldung: siehe 5.-6.2.

4.-5.11. - Göppingen: Praxis-Seminar: Diagnostik und Therapie des Rheumaschmerzes. Anmeldung: siehe 29.-30.4.

11.-12.11. - Berlin: Praxis-Seminar: Aktueller Stand der Schmerztherapie in Klinik und Praxis. Anmeldung: siehe 5.2.

26.11. - Bad Homburg: Festveranstaltung: Schmerz und Lebensqualität. III. Verleihung des Deutschen Förderpreises für Schmerzforschung und Schmerztherapie. Anmeldung: siehe 18.-19.3.

2.-3.12. - Bad Schwartau: Praxis-Seminar: Rationelle Therapie der Migräne. Anmeldung: siehe 24.2.

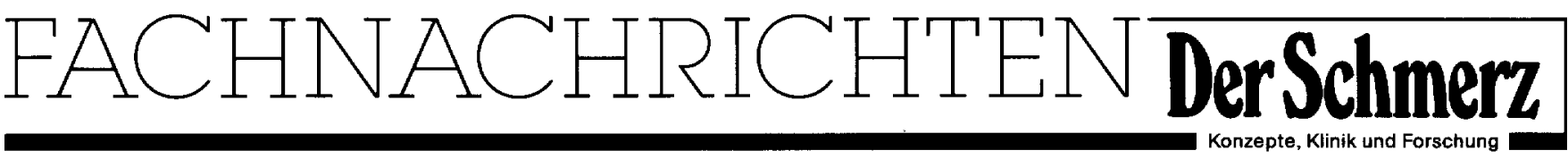

\section{Ausschreibung des Sertürner-Preises}

Aus Anlaß des 200. Geburtstages des Apothekers Friedrich Wilhelm Sertürner, dem Entdecker des Morphiums, verleiht die Sertürner Gesellschaft e.V., Einbeck, jährlich den Sertürner-Preis für die beste Arbeit über wissenschaftliche $\mathrm{Er}$ kenntnisse auf dem Gebiet ,Physio- logie, Pathophysiologie, Pharmakologie und Therapie des Schmerzes". Der Preis ist gestiftet von der Firma Smith Kline Dauelsberg, Göttingen/München und ist mit DM 10000, - dotiert. Er wird im Rahmen einer wissenschaftlichen Tagung in Einbeck vergeben. Die
Arbeiten müssen bis zum 15. April des jeweiligen Jahres in deutscher oder englischer Sprache eingereicht werden. Über die Bedingungen der Preisvergabe erteilt Auskunft und Einsendungen sind zu richten an: Prof. Dr. A. Doenicke, Institut für Anästhesiologie der LMU, Bereich Poliklinik, Pettenkoferstraße 8a, D-8000 München 2.

weils bis zum 1. September eines jeden Jahres erbeten an die Abteilung für Anästhesie, Intensivmedizin und Schmerztherapie, RotesKreuz-Krankenhaus, St.-PauliDeich 24, D-2800 Bremen 1. Auskunft über die Verleihungsbestimmungen erteilt Gh. Sehhati-Chafai, Bremen.

rechte Spalte, im 1. Satz heißt es irrtümlicherweise, daß die Obstipation als Nebenwirkung von Buprenorphin nicht in der Produktinformation der Herstellerfirma genannt sei. Tatsächlich ist jedoch dieser Hinweis in der Zwischenzeit dort aufgenommen worden.
Schmerz (1987) 1:48-51, Beitrag „Klinische Erfahrungen mit Buprenorphin" von M. Zenz. Auf S. 48, tacks before treatment included nausea (18), vomiting (9), and tophobia (19) but these were completely relieved by AH 25086 B on all occasions.

Doenicke und E. Siegel. Auf S. 29 muß der Satz im Abstract, Zeile 16, richtig lauten: Accompanying clinical symptoms in the migraine at- mit DM 5000, - dotiert ist und für Schmerzdiagnostik und -therapie verliehen wird. Der Preis ist limiJahren (1987 bis 1997). Einsendungen von Manuskripten werden je-

Verantwortlich für den Textteil: Prof. Dr. H. Bergmann, Ludwig-Boltzmann-Institut für experimentelle Anaesthesiologie und intensivmedizinische Forschung Wien-Linz, Bereich Linz, Krankenhausstraße 9, A-4020 Linz, Prof. Dr. M. Zimmermann, II. Physiologisches Institut der Universität, Abteilung Physiologie des Zentralnervensystems, Im Neuenheimer Feld 326, D-6900 Heidelberg

Für den Anzeigenteil: E. Lückermann, B. Völker, Heidelberger Platz 3, D-1000 Berlin 33

Gesamtherstellung der Universitätsdruckerei H. Stürtz AG, D-8700 Würzburg. Printed in Germany. O Springer-Verlag Berlin Heidelberg 1987

Springer-Verlag GmbH \& Co KG, D-1000 Berlin 33

Dieses Heft enthält je eine Beilage der Firmen Schwa-Medico GmbH., Frankfurt und 3-M Deutschland GmbH., Neuss 